Check for updates

Cite this: RSC Adv., 2018, 8, 25051

\title{
In situ synthesis of metal embedded nitrogen doped carbon nanotubes as an electrocatalyst for the oxygen reduction reaction with high activity and stability $\dagger$
}

\begin{abstract}
Yanhong Yin, (D) abc Hengbo Zhang, ${ }^{\text {abc }}$ Rongzhen Gao, ${ }^{\text {abc }}$ Aili Wang, ${ }^{\text {abc }}$ Xinxin Mao, ${ }^{a b c}$ Hongyu Dong ${ }^{\mathrm{abc}}$ and Shuting Yang (D) *abc

In this work, a Co-N doped carbon nanotube (CNT) catalyst was fabricated via a simple pyrolysis approach and the effects of solvothermal processing on the catalytic activity of the as-prepared material were investigated in detail. The results show that after solvothermal processing (Co-NC) the catalyst has a more homogeneous anemone structure, a higher nitrogen content, a larger BET surface area and a higher degree of graphitization compared to the catalyst produced after non-solvothermal processing (Co-MA). The results of electrochemical tests indicate that Co-NC, compared to commercial $20 \% \mathrm{Pt} / \mathrm{C}$ and Co-MA, has an improved mass transfer process and sufficient active site exposure, which brings about superb oxygen reduction electrocatalytic activity, a higher reduction potential $(-0.2 \mathrm{~V} v \mathrm{vs}$ Ag/ $\mathrm{AgCl})$, a limiting diffusion current $\left(5.44 \mathrm{~mA} \mathrm{~cm}^{-2}\right)$ and excellent stability in $0.1 \mathrm{M} \mathrm{KOH}$ solution.
\end{abstract}

Received 12th April 2018 Accepted 4th June 2018

DOI: 10.1039/c8ra03139h

rsc.li/rsc-advances
A common method to fabricate $\mathrm{M} / \mathrm{CNTs}$ is to prepare CNTs firstly via arc-discharge evaporation ${ }^{\mathbf{1 5}}$ or a chemical vapor deposition (CVD) method under a catalyst such as Fe, Co or Ni. To obtain pure CNTs, the products have to undergo an acid washing process to get rid of the redundant catalyst. Then, a solution reduction process is used to load metal nanoparticles on the as-prepared CNTs. However, CNTs with Fe or Co nanoparticles have proved to be an excellent catalyst for the ORR process. So it is efficient to prepare an excellent M/CNT catalyst in one step.

Research has shown that dopants and defects can alter the properties of carbon. In particular, nitrogen doped carbon can greatly modulate carbon's surface properties, and increase the active sites and electrical conductivity of the pristine material, thus significantly enhancing its electrochemical performance. ${ }^{16,17}$ Chen et al. ${ }^{18}$ synthesized a series of $\mathrm{Co} / \mathrm{N} / \mathrm{C}$ catalysts via pyrolysis of a mixture of cobalt acetate and a prepolymer of melamine formaldehyde resin. Similarly, Fe/N/C hollow nanospheres were obtained via an Fe(III)-dopamine-complexationassisted one-pot method. ${ }^{19}$ Furthermore, embedded cobalt nanocrystals in the graphene catalysts prepared by a single-step approach showed that their electro-catalytic properties outperform those of $\mathrm{Pt} / \mathrm{C}^{20}$

The research in highly cost effective transition metal nitrogen doped-carbon ORR catalysts depends heavily on catalyst design and synthesis innovation. Commonly, a pyrolysis strategy is used to prepare the catalysts, during which a mixture containing a nitrogen precursor, carbon precursor and transition metal undergoes heat processing to obtain the desired
Henan 453007, P. R. China

${ }^{b}$ National \& Local Joint Engineering Laboratory for Motive Power and Key Materials Xinxiang, Henan 453007, China

${ }^{c}$ Collaborative Innovation Center of Henan Province for Motive Power and Key Materials, Xinxiang, Henan 453007, China

$\dagger$ Electronic supplementary information (ESI) available. See DOI: 10.1039/c8ra03139h 
product. ${ }^{19,21}$ In previous work, much emphasis has been given to metal species, $\mathrm{N}$ and $\mathrm{C}$ resources, templates, supports and pyrolysis conditions, but little attention has been paid to precursor processing. As is well known, the structure and morphology of the precursor will affect greatly the final performance of the catalyst. Therefore, this study makes a major contribution to research on the effect of different precursor processing strategies by comparing the physical structure and electrochemical properties.

Here, using melamine, glyoxal and cobalt nitrate hexahydrate as raw materials, Co-N doped carbon nanotube (CNT) catalysts are synthesized via a simple in situ pyrolysis method. Melamine is the ideal source for $\mathrm{N}$ and $\mathrm{C}$, and its three symmetrical amino-groups can react with glyoxal or complex with cobalt under solvothermal conditions, which is a benefit for the uniform distribution of the cobalt element. Emphasis is given in the study as to whether and how solvothermal processing affected the catalytic performance of the Co-NC material.

\section{Results and discussion}

The schematic diagram for synthesizing Co-NC is shown in Fig. 1. Glyoxal, melamine and cobalt-nitrate hexahydrate were first mixed uniformly. During the solvothermal reaction, amide groups formed between melamine and glyoxal, and resulted in the formation of spherical shell or flake structures. At the same time, cobalt-nitrate hexahydrate can complex with $-\mathrm{NH}_{2}$ from melamine. Due to the coordination of cobalt ions, the spherical shells or flakes curl into a tubular structure. This can be proved using the images shown in Fig. S1 and S2. $\dagger$ The morphology of the Co-free precursor (named Co-freeP) displays a micro-sphere or flake structure (Fig. S1a $\dagger$ ) while that of the Co-NC precursor (named Co-NCP) shows a tubular structure (Fig. S1b†). After the pyrolyzing process, they transform into cystoid and anemone structures, respectively (Fig. S2 $\dagger$ ). From the FT-IR results (Fig. S3†), one can observe a band at $3215-3353 \mathrm{~cm}^{-1}$ from CofreeP and Co-NCP which indicates the stretching vibration of $\mathrm{HN}-\mathrm{C}-\mathrm{NH}{ }^{22}$ The two small peaks with a tag in Fig. S3† correspond to the asymmetric stretching vibration of the $\mathrm{HN}-\mathrm{C}-\mathrm{NH}$ bond, which proves that melamine and glyoxal might participate in the chemical reaction and the formation of amide groups. ${ }^{22}$ For Co-NCP, a stretching vibration of the Co- $\mathrm{N}_{x}$ bonds (2412 $\mathrm{cm}^{-1}$ ) can be observed, inferring that complexation between melamine and cobalt-nitrate has occured. ${ }^{23}$

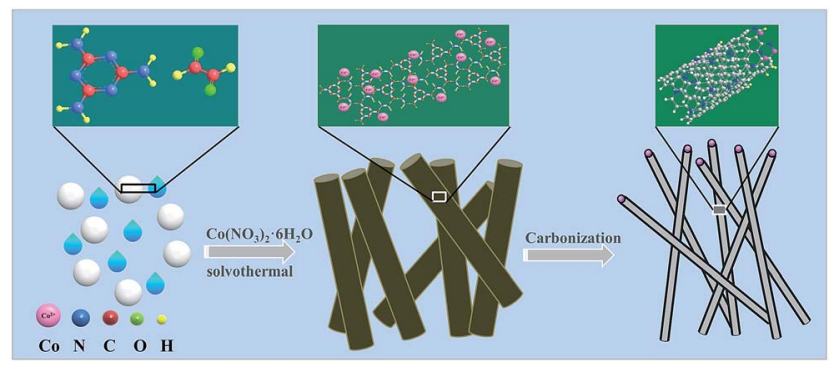

Fig. 1 Schematic drawing for the synthetic procedure of Co-NC.
The morphologies of Co-NC and Co-MA are shown in Fig. 2. It was found that Co-NC grows as relatively uniform carbon nanotubes of several micrometers long and 50-150 nm thick (Fig. 2a) while non-uniform nanotubes and nano-particles are observed for Co-MA (Fig. 2b). It is possible that the solvothermal process benefits the uniform distribution of $\mathrm{Co}$, and as a result, the carbon nanotubes formed will be more uniform. ${ }^{24}$ The microstructure of Co-NC is further studied by TEM and HR-TEM analysis. Fig. 2c clearly shows images of in situ grown CNTs and the presence of another constituent in the head of the CNTs. The HR-TEM image of the end of the carbon nanotube is shown in Fig. 2d. Lattice fringes of the inner core are consistent with crystalline Co with an interspacing of $0.204 \mathrm{~nm}$. The lattice spacing of the outer shell is $0.35 \mathrm{~nm}$, which is in accordance with that of graphitic carbon. ${ }^{25}$ This result reveals that the crystalline Co nanoparticles were embedded in highly graphitic carbon which was composed of a few graphite layers.

The existence of graphitic carbon and cobalt element in the as-prepared Co-NC and Co-MA samples can also be verified by XRD analysis as shown in Fig. 3a. Both of them exhibit three distinct peaks at $51.5^{\circ}, 44.2^{\circ}$ and $75.8^{\circ}$ which demonstrates a crystal face homologous to that of metallic Co (111), (200) and (220), respectively. This result indicates that $\mathrm{Co}^{2+}$ in the precursor has been reduced to metallic Co by the reducing gases produced in the pyrolysis step. The peaks at $26.3^{\circ}$ in the inset confirm the existence of graphitic carbon.

Fig. 3b shows the Raman spectra of Co-NC and Co-MA, which display the characteristic Raman shifts of CNTs. The intensity of the D peak at $1351 \mathrm{~cm}^{-1}$ is correlated to the disordered state of carbon. The intensity of the $\mathrm{G}$ peak $\left(1588 \mathrm{~cm}^{-1}\right)$ is correlated to the degree of graphitization of carbon. ${ }^{26} I_{\mathrm{D}} / I_{\mathrm{G}}$ is often applied to measure the degree of graphitization. For Co$\mathrm{NC}$ and Co-MA, the $I_{\mathrm{D}} / I_{\mathrm{G}}$ values fitted using Origin are 1.32 and 1.96, respectively. This indicates that the Co-NC catalyst has a relatively higher graphitization degree compared with that of Co-MA. As is well known, $\mathrm{Co}^{2+}$ can catalyze the graphitization of carbon. ${ }^{27}$ The uniform distribution of Co helps enhance graphitization, ${ }^{18}$ which helps increase the stability of electrocatalysts.

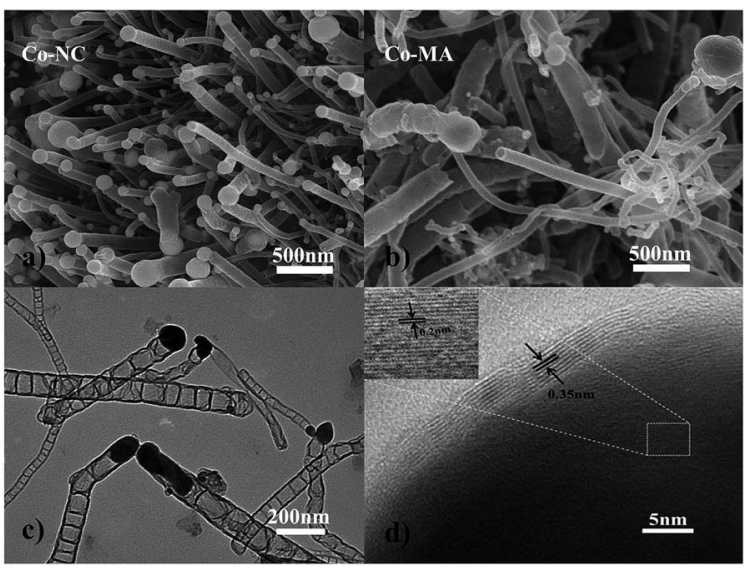

Fig. 2 SEM images of cobalt embedded in a nitrogen-doped nanotube catalyst: (a) Co-NC and (b) Co-MA. A TEM image (c) and HR-TEM image (d) of Co-NC. 

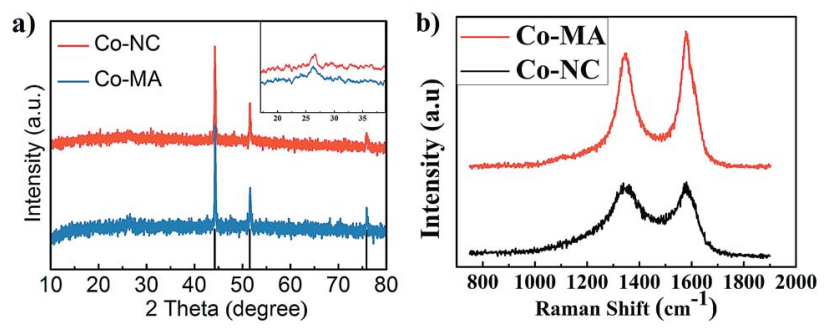

Fig. 3 (a) XRD patterns of Co-MA and Co-NC; the inset shows the carbon characteristic peak. (b) The Raman spectra of Co-MA and CoNC.

A survey of the chemical construction of Co-NC and Co-MA was carried out by XPS analysis (Fig. 4 and S4 $\dagger$ ). Co, N and $\mathrm{C}$ characteristic peaks are all observed, as indicated in the XPS survey spectrum in Fig. 4a. Fig. 4b shows the $\mathrm{C}$ spectrum. Two major narrow peaks at $284.0 \mathrm{eV}$ and $284.85 \mathrm{eV}$ can be observed, which belong to $\mathrm{C}-\mathrm{C}$ and $\mathrm{C}-\mathrm{OH}$, respectively. According to the previous conclusion, the presence of $\mathrm{C}-\mathrm{OH}$ groups could ensure that the carbon nanotubes are hydrophilic. ${ }^{28}$ It is well known that hydrophilicity could improve the migration rate of reactants. This ensures that the dissolved oxygen is more accessible to the catalyst.

As shown in Fig. 4c, the Co 2p spectrum displays five major narrow peaks at $781.5 \mathrm{eV}, 785.9 \mathrm{eV}, 797.3 \mathrm{eV}$ and $802.9 \mathrm{eV}$. The intense peaks at $785.9 \mathrm{eV}$ and $802.9 \mathrm{eV}$ are assigned as satellite peaks that could be attributed to high-spin $\mathrm{Co}^{2+}$ ions. The intense peak at $781.5 \mathrm{eV}$ is consistent with $\mathrm{Co}-\mathrm{N}_{x},{ }^{29}$ while that at around $797.3 \mathrm{eV}$ is ascribed to the $\mathrm{Co}_{3} \mathrm{O}_{4}$ phase. ${ }^{30} \mathrm{Co}^{0}$ peaks cannot be found, which may be because of cobalt oxide on the surface. In addition, as expected, the $\mathrm{C}$ spectrum and Co $2 \mathrm{p}$ spectrum of Co-MA (Fig. 4Sc $\dagger$ ) show the same features as those of Co-NC.

Fig. 4d shows the high-resolution $\mathrm{N}$ 1s spectrum of Co-NC. As is well known, the oxygen reduction catalytic ability is related to the amount and species of nitrogen. ${ }^{31}$ The existence of $\mathrm{N}$ indicates that $\mathrm{N}$ has been incorporated into the carbon matrix. Herein, we show the different types of $\mathrm{N}$-doping for Co-
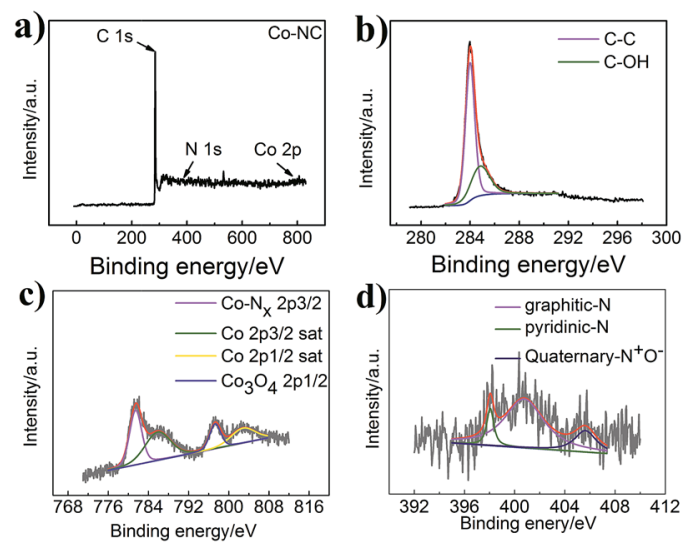

Fig. 4 (a) XPS spectrum of Co-NC, (b) the deconvoluted C1s spectrum of Co-NC, (c) the deconvoluted Co2p spectrum of Co-NC, and (d) the deconvoluted N1s spectrum of Co-NC.
NC (5.48\%) and Co-MA (3.36\%). For Co-NC, there are four peaks at $404.6 \mathrm{eV}, 401.2 \mathrm{eV}, 399.1 \mathrm{eV}$ and $398.7 \mathrm{eV}$, which are in accordance with quaternary- $\mathrm{N}^{+} \mathrm{O}^{-}$, graphitic- $\mathrm{N}$, Co- $\mathrm{N}_{x}$ and pyridinic-N. Meanwhile, for Co-MA (Fig. S4d $\dagger$ ), there are only three peaks at $405.64 \mathrm{eV}, 400.75 \mathrm{eV}$ and $398.05 \mathrm{eV}$ which correspond to quaternary- $\mathrm{N}^{+} \mathrm{O}^{-}$, graphitic- $\mathrm{N}$ and pyridinic- $\mathrm{N}$. The atom ratios of the different types of $\mathrm{N}$ are shown in Table 1(ESI $\dagger$ ). According to the literature, ${ }^{32}$ the high pyridine-N content of Co-NC is helpful for the enhancement of ORR catalytic activity. ${ }^{33}$

The results of the analysis of pore structure and specific surface area $\left(S_{\mathrm{BET}}\right)$ are shown in Fig. 5 and the detailed data of these materials are displayed in Table 2 (ESI $\dagger$ ). Both of them demonstrate type IV isotherms, and the hysteresis loops can be observed at $0.5-1.0 P / P_{0}$, which indicates the porous structure of the materials. Here, the pore size and the calculated results about pore distribution obtained via the Barrett-JoynerHalenda (BJH) method are shown in Fig. 5b. One can find that both of the samples mainly have two kinds of pore size distribution: one is 1-10 $\mathrm{nm}$ (mesopores) and the other is 100$150 \mathrm{~nm}$ (macropores). From Table 2(ESI $\dagger$ ), the BET surface area of Co-NC and Co-MA is almost the same, but the former has a relatively larger pore size and pore volume than those of the latter, which can facilitate reactant transportation inside the material.

Fig. 6a shows the CV curves of commercial $20 \mathrm{wt} \% \mathrm{Pt} / \mathrm{C}$ (JM) and the above-mentioned materials that were obtained despite the capacitive background current. The potentials mentioned here are all measured vs. a $\mathrm{Ag} / \mathrm{AgCl}$ reference electrode. A standard ORR peak of Co-NC appeared at ca. $-0.21 \mathrm{~V}$ in the $\mathrm{O}_{2}$ saturated electrolyte, which is inferior to that of $\mathrm{Pt} / \mathrm{C}(0.095 \mathrm{~V})$ but higher than that of Co-MA $(-0.25 \mathrm{~V})$. The results show that Co-NC is more effective for the ORR process.

Fig. $6 \mathrm{~b}$ shows the results of the LSV experiments of the three samples. Onset potentials are acquired from a rotating disk electrode under a linear sweep of $1600 \mathrm{rpm}$. From Fig. 6b, one can find that the onset potential of Co-NC is $-0.132 \mathrm{~V}$, which is slightly higher compared to that of Co-MA $(-0.168 \mathrm{~V})$. On the other hand, Co-NC appears to have a much higher limiting current density than that of the $\mathrm{Pt} / \mathrm{C}$ and Co-MA catalysts. Linear sweep voltammetry (LSV) curves of Co-NC from 400 to $2025 \mathrm{rpm}$ and the $\mathrm{K}-\mathrm{L}$ curves with a closely paralleled dependency are shown in Fig. $6 c$ and the inset. To investigate the reaction process of the catalyst surface, the transferred electron number $(n)$ was calculated using eqn (1) and (2) and the results

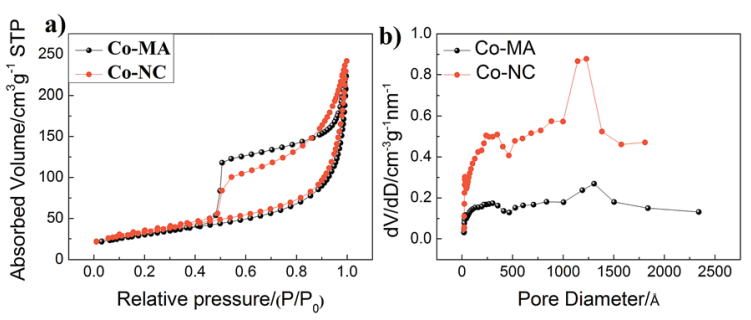

Fig. 5 (a) The $\mathrm{N}_{2}$-sorption isotherm curve and (b) pore distribution of Co-NC and Co-MA samples. 

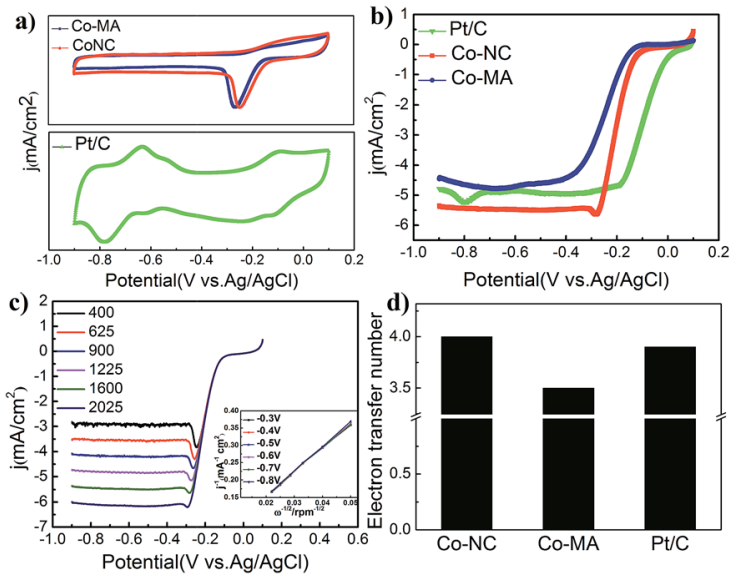

Fig. 6 (a) CV curves of Co-NC, Co-MA and Pt/C under a $50 \mathrm{mV} \mathrm{s}^{-1}$ scan rate, (b) LSV curves under a $10 \mathrm{mV} \mathrm{s}^{-1}$ scan rate at $1600 \mathrm{rpm}$, (c) $\mathrm{K}-\mathrm{L}$ curves and LSV curves of Co-NC determined using a RDE under a $10 \mathrm{mV} \mathrm{s}^{-1}$ scan rate (at $400 \mathrm{rpm}, 625 \mathrm{rpm}, 900 \mathrm{rpm}, 1225 \mathrm{rpm}$, $1600 \mathrm{rpm}$ and $2025 \mathrm{rpm}$ ), and (d) a histogram of the transferred electron number $(n)$ of Co-NC, Co-MA and Pt/C.
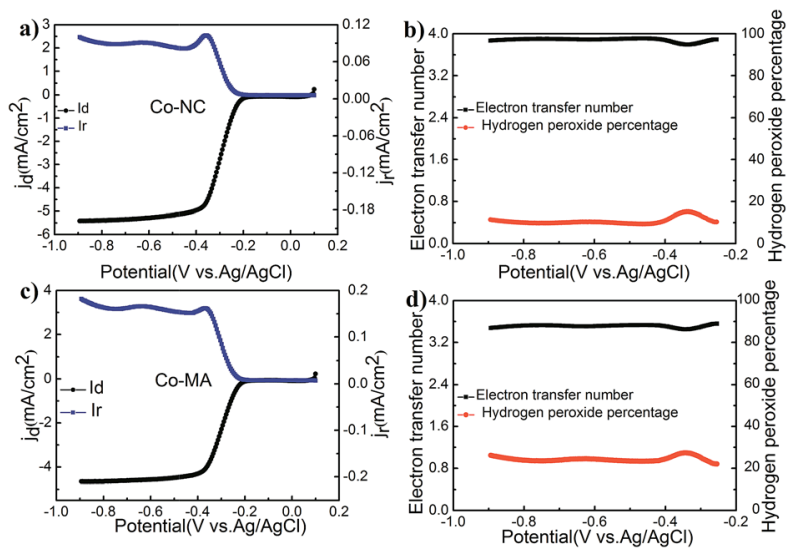

Fig. 7 (a) $I_{D}$ and $I_{R}$ of Co-NC from RRDE experiments under a $10 \mathrm{mV}$ $\mathrm{s}^{-1}$ scan rate, (b) $n$ and $\mathrm{HO}_{2}{ }^{-} \%$ content of Co-NC, (c) $I_{\mathrm{D}}$ and $I_{\mathrm{R}}$ of CoMA from RRDE experiments under a $10 \mathrm{mV} \mathrm{s}^{-1}$ scan rate, and (d) $n$ and $\mathrm{HO}_{2}{ }^{-} \%$ content of Co-MA.

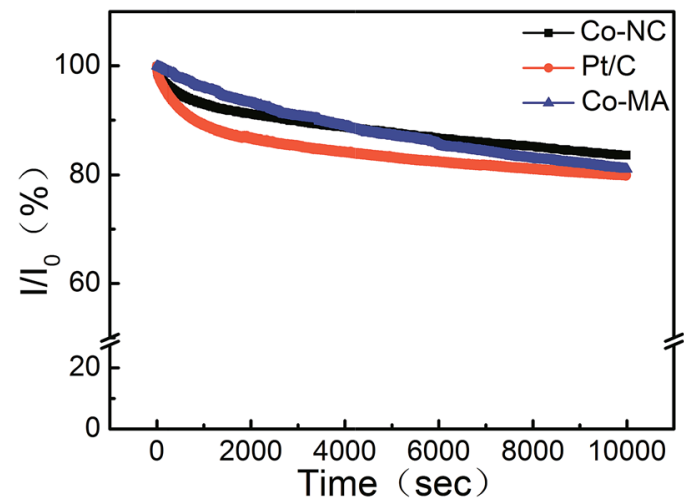

Fig. 8 Chronoamperometry curves at $-0.2 \mathrm{~V}$ of Co-NC, Co-MA and $\mathrm{Pt} / \mathrm{C}$ under $1600 \mathrm{rpm}$. are shown in Fig. 6d. The transferred electron number of the CoNC electrode is approximately 4.0, which is higher than that of Co-MA (3.5), suggesting that the catalytic process on the Co-NC surface progressed via a four-electron approach in which $\mathrm{O}_{2}$ was reduced to $\mathrm{H}_{2} \mathrm{O}$ directly. This four-electron catalytic approach can increase reaction speed compared to the two-electron twostep approach. The results about $\mathrm{M} / \mathrm{NC}$ catalysts in the literature are summarized in Table 3 (ESI $\dagger$ ). Compared with other M/ $\mathrm{NC}$ catalysts, the Co-NC catalyst in this work shows the highest limiting diffusion current $\left(J_{\text {limit }}\right)$ and transferred electron number $(n)$.

RRDE technology is an effective tool to study this oxygen reduction process. Fig. 7 shows the results of an RRDE test. The transferred electron numbers $(n)$ of $\mathrm{Co}-\mathrm{NC}$ and Co-MA under different potentials were determined using eqn (3) and the main values were close to those obtained from using the RDE technique. One can also find that the Co-NC electrode has a relatively lower $\mathrm{HO}_{2}{ }^{-} \%$ content, inferring that the catalytic reaction is a four electron process. The excellent catalytic activity of Co$\mathrm{NC}$ may be due to the relatively higher $\mathrm{N}$ content and the Co- $\mathrm{N}_{x}$ structure. $^{34}$

The durability of Co-NC and Co-MA was investigated by measuring the chronoamperometric (CA) response in comparison to that of $\mathrm{Pt} / \mathrm{C}$ at $-0.2 \mathrm{~V}$ in $\mathrm{KOH}$ solution $\left(0.1 \mathrm{M}, \mathrm{O}_{2}-\right.$ - saturated). After $10000 \mathrm{~s}$, the effective current densities on CoNC, Co-MA and Pt/C decreased by $16.4 \%, 18.9 \%$ and $20.2 \%$, respectively. This indicates that the ORR active site washed away in alkaline medium. The small current density loss of Co-NC may be due to the enhanced degree of graphitization of Co$\mathrm{NC},{ }^{35}$ which is a benefit from the uniform distribution of Co after the solvothermal process (Fig. 8).

\section{Conclusions}

In summary, Co-N doped carbon nanotube (CNT) catalysts have been synthesized via a simple in situ pyrolysis method and the effect of solvothermal processing on the catalyst precursors has been examined. Results confirm that the catalysts fabricated by solvothermal processes, compared to that by non-solvothermal processes, have more homogeneous morphology, larger pore size and pore volume, higher pyridine-N content (48.1\%) and a Co- $\mathrm{N}_{x}$ structure, which leads to excellent stability and catalytic activity in alkaline medium. This work mainly focus on the effects of different precursor processes, and these discoveries will help us to optimize synthesis methods for $\mathrm{M} / \mathrm{N} / \mathrm{C}$ catalysts.

\section{Experimental}

\section{Materials}

Melamine and glyoxal solution (40\%) were obtained from Sinopharm Chemical Reagent Co., Ltd. Cobalt nitrate hexahydrate was purchased from Shanghai Macklin Biochemical Co., Ltd. First, $5 \mathrm{~g}$ melamine and $3.5 \mathrm{~g}$ cobalt nitrate hexahydrate were added into $20 \mathrm{ml}$ alcohol with magnetic stirring. After ultrasonic dispersion for $15 \mathrm{~min}, \mathrm{NaOH}$ solution was used to alter the $\mathrm{pH}$ values. $2.5 \mathrm{ml}$ glyoxal solution was dropped in the suspension under continuous magnetic stirring. This 
suspension was transferred into a high pressure reactor, and ethanol was added to make it $80 \%$ full. After heating the reactor at $180^{\circ} \mathrm{C}$ for $4 \mathrm{~h}$, the product was air-dried at room temperature, and then heated to $900{ }^{\circ} \mathrm{C}$ for $2 \mathrm{~h}$ in $\mathrm{N}_{2}$ in a tube furnace. The final product was named Co-NC. To explore the formative process of Co-NC, the metal-free catalyst was synthesized using the same method as Co-NC but cobalt nitrate was not added, and it was named Co-free.

In contrast, another sample was prepared under the same conditions as Co-NC except for the fact that the precursors didn't undergo the solvothermal process. This as-prepared sample was denoted Co-MA.

\section{Characterization}

The physical structure of the above-mentioned samples was studied using a field emission scanning electron microscope (FESEM, JSM-6700F) and a transmission electron microscope (TEM, JEM 2100), with an accelerating voltage of $15 \mathrm{kV}$ and 200 $\mathrm{kV}$ respectively. The structure and constituents of the samples were represented by carrying out X-ray diffraction (XRD) on a D8 Advance (Bruker) diffractometer with $\mathrm{Cu} \mathrm{K} \alpha$ radiation. X-ray photoelectron spectroscopy (XPS) was carried on an AXIS Ultra DLD system. The specific surface areas were assessed using a Micromeritics Tristar 3020 gas adsorption analyzer. Raman spectra of the powder samples were collected using a Lab-RAM HR800 instrument. Fourier transform infrared spectra (FT-IR) were measured using a NEXUS spectrophotometer.

\section{Electrochemical measurements}

All of the tests were performed on a CHI 660B electrochemical workstation and a standard three-electrode system in $0.1 \mathrm{M}$ $\mathrm{KOH}$ solution was adopted. A glassy carbon electrode (GCE) was regarded as the working electrode, and we opted to use a $\mathrm{Ag}$ / $\mathrm{AgCl}$ electrode and platinum wire as the reference and counter electrode. For the modification of the working electrode, $5 \mathrm{mg}$ of catalyst and acetylene black respectively, $95 \mu \mathrm{L}$ $5 \mathrm{wt} \%$ Nafion solution and $350 \mu \mathrm{L}$ ethanol were mixed and dispersed homogeneously using an ultrasonic technique. Then this mixture was dropped onto the GCE and dried. The catalyst loading was $0.03 \mathrm{mg} \mathrm{cm} \mathrm{cm}^{-2}$. Prior to each measurement, the modified electrode was activated in the same chemical environment. The scan rate for RDE polarization curves was $0.01 \mathrm{~V}$ $\mathrm{s}^{-1}$ or $0.05 \mathrm{~V} \mathrm{~s}^{-1}$. Koutechy-Levich $(\mathrm{K}-\mathrm{L})$ curves were drawn using the following formulas:

$$
1 / j=1 / j_{\mathrm{k}}+1 / B \omega^{1 / 2}
$$

where $j_{\mathrm{k}}$ is the kinetic-limiting current density, $j$ is the measured current density and $\omega$ is the revolving speed. $B$ is a constant which relates to the slope of $\mathrm{K}-\mathrm{L}$ curve.

$$
B=0.2 n F C_{0} D_{0}^{2 / 3} \nu^{-1 / 6}
$$

where $D_{0}$ is the diffusion coefficient and $C_{0}$ is the bulk concentration of $\mathrm{O}_{2}$ in the $0.1 \mathrm{M} \mathrm{KOH}$ aqueous solution, $F$ is the Faraday constant (96 485 $\mathrm{C} \mathrm{mol}^{-1}$ ), $\nu$ is the kinematic viscosity of the electrolyte and $n$ is the electron transfer number which was calculated via the following equation:

$$
n=\operatorname{abs}\left(I_{\mathrm{D}}\right) /\left(\operatorname{abs}\left(I_{\mathrm{D}}\right) \times 4+\left(I_{\mathrm{R}} / N\right)\right)
$$

where $N$ is equal to 0.22 and means the disk electrode collection efficiency.

The $\% \mathrm{HO}_{2}{ }^{-}$was calculated based on eqn (4):

$$
\% \mathrm{HO}_{2}^{-}=100 \times\left(2 I_{\mathrm{R}} / N\right) /\left(\operatorname{abs}\left(I_{\mathrm{D}}\right)+\left(I_{\mathrm{R}} / N\right)\right)
$$

where $I_{\mathrm{R}}$ is the ring current and $I_{\mathrm{D}}$ the disk current which are all determined by carrying out RRDE measurements.

\section{Conflicts of interest}

There are no conflicts to declare.

\section{Acknowledgements}

This research work has been financially supported by the Plan For Scientific Innovation Talent of Henan Province (174100510015) and the National Nature Science Foundation of China (51502082).

\section{Notes and references}

1 M. K. Debe, Nature, 2012, 486, 43-51.

2 J.-H. Wee, Renewable Sustainable Energy Rev., 2007, 11, 17201738.

3 A. H. Brian and C. H. Steele, Mater. Sustainable Energy Appl., 2010, 224-231.

4 C. Giordano and M. Antonietti, Nano Today, 2011, 6, 366380.

5 Y. Hu, J. O. Jensen, W. Zhang, L. N. Cleemann, W. Xing, N. J. Bjerrum and Q. Li, Angew. Chem., Int. Ed., 2014, 53, 3675-3679.

6 N. Levy, A. Mahammed, M. Kosa, D. T. Major, Z. Gross and L. Elbaz, Angew. Chem., Int. Ed., 2015, 54, 14080-14084.

7 Q. Lu, M. Zhao, J. Chen, B. Chen, C. Tan, X. Zhang, Y. Huang, J. Yang, F. Cao, Y. Yu, J. Ping, Z. Zhang, X. J. Wu and H. Zhang, Small, 2016, 12, 4669-4674.

8 M. Q. Wang, C. Ye, S. J. Bao, Z. Y. Chen, Y. N. Yu, Y. Zhang and M. W. Xu, Chem. Commun., 2016, 52, 12992-12995.

9 B. Y. Xia, Y. Yan, N. Li, H. B. Wu, X. W. Lou and X. Wang, Nat. Energy, 2016, 1, 15006.

10 C. Tang, B. Wang, H. F. Wang and Q. Zhang, Adv. Mater., 2017, 29, 1703185.

11 D. Wang, Z. Ma, Y. e. Xie and H. Song, J. Energy Chem., 2017, 26, 1187-1195.

12 H. Wu, X. Jiang, Y. Ye, C. Yan, S. Xie, S. Miao, G. Wang and X. Bao, J. Energy Chem., 2017, 26, 1181-1186.

13 X. Chen, S. Sun, X. Wang, F. Li and D. Xia, J. Phys. Chem. C, 2012, 116, 22737-22742.

14 P. Z. Guo Gang, J. Am. Chem. Soc., 2013, 46, 1878-1889.

15 S. Iijima, Nature, 1991, 354, 56. 
16 C. Tang, M.-M. Titirici and Q. Zhang, J. Energy Chem., 2017, 26, 1077-1093.

17 C. Tang and Q. Zhang, Adv. Mater., 2017, 29, 1604103.

18 S. Z. Chen, W. Yang, L. W. Li, H. B. Zou and W. M. Lin, Adv. Mater. Res., 2012, 557-559, 1218-1222.

19 L. Y. Dan Zhou, L. Yu, J. Kong, X. Yao, W. Liu, Z. Xu and X. Lu, Nanoscale, 2014, 1-8.

20 L.-B. Lv, T.-N. Ye, L.-H. Gong, K.-X. Wang, J. Su, X.-H. Li and J.-S. Chen, Chem. Mater., 2015, 27, 544-549.

21 X. Zhang, R. Liu, Y. Zang, G. Liu, G. Wang, Y. Zhang, H. Zhang and H. Zhao, Chem. Commun., 2016, 52, 59465949.

22 C. Hu, Y. Xiao, Y. Zhao, N. Chen, Z. Zhang, M. Cao and L. Qu, Nanoscale, 2013, 5, 2726.

23 Inorganics IR Grating Spectra, 1972, vol. Y1k-Y600k, p. Y54k. 24 P. Lukanov, V. K. Anuganti, Y. Krupskaya, A.-M. Galibert,

B. Soula, C. Tilmaciu, A. H. Velders, R. Klingeler, B. Büchner and E. Flahaut, Adv. Funct. Mater., 2011, 21, 3583-3588.

25 C. Han, X. Bo, Y. Zhang, M. Li, A. Nsabimana and L. Guo, Nanoscale, 2015, 7, 5607-5611.
26 L. Cao, Z. Lin, J. Huang, X. Yu, X. Wu, B. Zhang, Y. Zhan, F. Xie, W. Zhang, J. Chen, W. Xie, W. Mai and H. Meng, Int. J. Hydrogen Energy, 2017, 42, 876-885.

27 X. Zou, X. Huang, A. Goswami, R. Silva, B. R. Sathe, E. Mikmeková and T. Asefa, Angew. Chem., Int. Ed., 2014, 53, 4372-4376.

28 G. S. P. Jang-Soo Lee, S. Tai Kim, M. Liu and J. Cho, Angew. Chem., Int. Ed., 2013, 52, 1026-1030.

29 E. A. K. I. N. Timonova, E. V. Boldyreva and L. N. Mazalov, J. Struct. Chem., 1988, 28, 68-76.

30 T. Y. Ma, S. Dai, M. Jaroniec and S. Z. Qiao, J. Am. Chem. Soc., 2014, 136, 13925-13931.

31 W. X. Justus Masa, M. Muhler and W. Schuhmann, Angew. Chem., Int. Ed., 2015, 54, 10102-10120.

32 R. S. Donghui Guo, C. Akiba, S. Saji and J. N. Takahiro Kondo, Science, 2016, 351, 361-365.

33 Y. W. Qi Shi, Z. Wang, Y. Lei, B. Wang, N. Wu, C. Han, S. Xie and Y. Gou, Nano Res., 2016, 9, 317.

34 M. Zeng, Y. Liu, F. Zhao, K. Nie, N. Han, X. Wang, W. Huang, X. Song, J. Zhong and Y. Li, Adv. Funct. Mater., 2016, 26, 4397-4404.

35 X. Wang, W. Li, Z. Chen, M. Waje and Y. Yan, J. Power Sources, 2006, 158, 154-159. 